\title{
The Role of Public Health in Disaster Preparedness, Mitigation, Response, and Recovery
}

\author{
Guest Editors: \\ Kimberley I. Shoaf, Dr PH ${ }^{1}$ and Steven J. Rottman, MD, FACEP ${ }^{2}$
}

1. Research Director, Center for Public Health and Disaster Relief, University of California-Los Angeles

2. Director, Center for Public Health and Disaster Relief, University of CaliforniaLos Angeles and President, World Association for Disaster and Emergency Medicine

Correspondence:

Kimberly Shoaf, Dr.PH

Center for Public Health and Disaster Relief

University of California-Los Angeles

Los Angeles, California 90024 USA

Keywords: assessments; characteristics; disasters; impact; interdisciplinary; management; media; mitigation; needs; preparedness; public health; recovery; resources; response

\section{Abbreviations: \\ UCLA = University of California-Los Angeles}

As populations grow and occupy spaces that are vulnerable to a variety of hazards, disasters will increase in frequency and impact. Many professional and scientific fields are involved in the study of disasters and the field of disaster science rapidly is becoming an interdisciplinary endeavor. While some disciplines have been involved in working together to reduce the impacts of hazards on the population for a long time, others are just beginning to join the fray. One relative newcomer to the field is health sciences. This is not to imply that medicine and public health have not been involved in the reduction of disasters, however their contributions have tended to occur in something of a professional isolation, not as part of the multidisciplinary disaster management and response team. Recently, however, there has been movement on both the part of the health sciences and the other disciplines to bridge the gap between the two groups. The UCLA Conference on Public Health and Disasters was an attempt to bring interdisciplinary groups together for three days of dialogue in order to stimulate further cooperative efforts to reduce the public health impacts of natural disasters.

One possible reason for the gap between health sciences professionals and the others involved in disaster planning and response is a lack of understanding of the critical roles that public health and other health professionals play in the disaster cycle. It is not uncommon for people to be unaware of what public health does, even in daily life, let alone in a disaster setting. So what is public health?

Public health is that branch of the health field that is responsible for protecting not individual health, but the health of the entire population. The role of public health is to ensure the conditions necessary for people to live healthy lives through community-wide prevention and protection programs. The fundamental obligations of agencies responsible for public health are to: 1) prevent epidemics and the spread of disease; 2) protect against environmental hazards; 3) prevent injuries; 4) promote and encourage healthy behaviors and mental health; 5) respond to disasters and assist communities in recovery; and 6) assure the quality and accessibility of health services.

The field of public health is in itself, an interdisciplinary field. Contributions from sociology, psychology and other behavioral sciences combine with the clinical perspectives from medicine, nursing, dentistry, and nutrition. Add in the skills of planning, administration, and policy studies combined with journalism and the rigor of biostatistics and epidemiology, and the result is a comprehensive, interdisciplinary team that has the theories, methods, and skills necessary to address the broad ranging obligations previously described.

The papers in this special theme issue of Prehospital and Disaster Medicine (PDM) were presented at the University of California-Los Angeles (UCLA) Conference on Public Health and Disasters, convened in April, 1999. They reflect the many diverse contributions and responsibilities of public health to disaster prevention, mitigation, preparedness, response, and recovery, and represent three different perspectives: 1) the theoretical viewpoint of the academician; 2) results of research; and 3) the practical experiences of those practicing in the field. Many of the articles incorporate all of these perspectives. Because of the multidisciplinary nature of disaster public health manuscripts in this issue of PDM, we thought it would be useful to you for us to frame the range of topics in these introductory remarks.

The first set of papers provides an 
overview of the public health impacts of natural and man-made disasters and the role of public health agencies in responding to these events. Eric Noji presents a summary of the impacts of disasters on the population's health. He emphasizes the need for the use of sound epidemiologic principles in the scientific study of the impacts of disasters. The consequences of disasters vary greatly, not only between disasters caused by different hazards, but also within a single category. Cultural, sociologic, economic, geologic, atmospheric, and demographic characteristics contribute to these unique impacts. As Noji states, "the overall objective(s) of disaster management (are) to assess the needs of disaster-affected populations, match resources to needs efficiently, prevent further adverse health effects, evaluate relief program effectiveness, and plan for future disasters."

Following this academic review of the public health impacts of disasters, Dave Abbott presents similar information from the perspective of an individual within a state health department responsible for disaster response. In recent years, the state of California has confronted a large number of disasters, and Abbott's experience in those responses provides an example of the skills and knowledge needed in state and local health departments.

One of the main tasks for public health agencies following a disaster is identifying the extent of adverse health impacts upon the affected population. Of the many possible methods described in the literature, one strategy that has been adapted very effectively for use in the immediate post-disaster period is a form of rapid health assessment. In her manuscript, Malilay describes this methodology, which is based on a technique originally used to assess the extent of immunization coverage in developing countries. The serial application of this rapid assessment survey can help the emergency management teams determine segments of the population most in need of restoration of critical resource, and evaluate the success of their interventions. In addition to basic health, sanitation, water, and shelter needs, a considerable portion of the population depends upon some form of health aid (i.e., eyeglasses, contact lenses, hearing aids, etc.) or prescription medication. The need to replace these items in the post-impact period can put a strain on health care resources, and this often unrecognized problem is described in the paper by Sareen.

In addition to the substantial impact that disasters can have on the physical health needs of a population, disasters also place strain on the emotional needs of a population. While the fact that disasters have an impact on a population's emotional health is widely recognized, the extent and nature of those effects are debated widely in the current literature. Kathleen Tierney addresses both sides of this debate and offers insight into why so widely divergent views are held by different segments of the disaster response community.

Public health agencies should not be addressing the health impacts of disasters in a vacuum. There are a number of other resources that can be partners in ameliorating the effects of disasters, and one of the most visible of these is the news media. But in order to work effectively with both the broadcast and print media, public health professionals need to understand the media's perspective on the disaster and their perceived role in reporting these events to the public. Two articles in this issue provide an overview of the media's point of view. The first, by Ball-Rokeach and Loges, describes from a theoretical stance, how public health can interact with the media before, during, and after a disaster, with emphasis on community-based print media. The second article by Terri Anzur provides the perspective of an individual on the frontlines of providing broadcast media coverage. Her manuscript describes the experience of a television news anchor covering a disaster, and the reactions of public health professionals to that coverage. She provides suggestions from a news anchor's perspective as to how public health officials might position themselves to be heard by the media.

Other potential partners for public health agencies in responding to disasters include volunteers, both as individuals and as a part of volunteer organizations, and we have included two manuscripts addressing this important community resource. Based on his research on emergent volunteers in disaster situations, David Simpson provides recommendations that would improve the value and utility of volunteers to the public health efforts in a disaster situation. Following this paper, Dusty Bowenkamp provides a "view from the trenches." In her manuscript, she describes the efforts that the Red Cross and other volunteer agencies have undertaken to respond to the health needs of populations affected by disasters. With more than 20 years of experience in Red Cross Disaster Services, Bowenkamp emphasizes the need for collaboration between volunteer and governmental agencies.

The issue is wrapped up by an article by Kenneth Kizer. He reinforces the concept of more and worse disasters to come, identifying the converging variables of an ever-increasing global population with serious threats to the stability of our environment, technological and biomedical innovations that could lead to large scale human hazards, a shrinking pool of health care resources relative to the increased need for these resources, and the emerging threat of bioterrorism. Kizer has, as he states, "had the opportunity to see public health emergency management as a front line responder, planner, policymaker, and investigator." His 10 "lessons learned" emphasize the important policy and practical applications of many of the points made by his colleagues whose manuscripts precede his.

As you can see, a number of critical issues are raised in this issue of PDM, covering a range of topics that are relevant to the field of disaster public health. It is vital that those who plan for and respond to large scale mass population health emergencies consider these perspectives and work to become an integrated part of the disaster management community. The disaster management table already is set. It is up to us in the health sciences community to decide if we are going to take our seat along with our colleagues from other fields, or continue to sit at a 
separate table. If we don't join the other participants, we will perpetuate a disjointed response, resulting in separate, isolated activities by EMS, hospitals, and municipal fire, utilities, and law enforcement agencies on the one hand, and the immediate- and long-term mass population health and social service concerns on the other. 